\title{
Iliopsoas haematoma in a rugby player
} \author{
A Jansen van Rensburg, ${ }^{1,2} \mathrm{MSc}$; C C Grant, ${ }^{1,2} \mathrm{PhD}$ \\ ${ }^{1}$ Section Sports Medicine, Faculty of Heath Sciences, University of Pretoria, South Africa \\ ${ }^{2}$ Exercise SMART Team, University of Pretoria, South Africa \\ ${ }^{3}$ High Performance Centre, University of Pretoria, South Africa \\ ${ }^{4}$ Blue Bulls Rugby Union, Pretoria, South Africa \\ ${ }^{5}$ Little Company of Mary Medical Centre, Pretoria, South Africa
}

D C Janse van Rensburg, ${ }^{1,2}$ MD, PhD, FACSM; O Strauss, ${ }^{1,3,4}$ MB ChB, MSc (Sports Med); M D Velleman, ${ }^{1,5}$ MMed (RadD), FCRad(D);

Corresponding author: D C Janse van Rensburg (christa.jansevanrensburg@up.ac.za)

Traumatic iliopsoas haematoma is a serious complication of haemorrhage disorders rarely seen in young healthy athletes. It is mostly described in patients on anticoagulant therapy and commonly associated with various degrees of femoral nerve palsy. A 22-year-old male rugby player presented with severe onset of pain in the lower back, right hip flexor/pelvic area following a tackle during a rugby match. Magnetic resonance imaging identified a distinct, hyperechoic heterogeneous mass within the right iliopsoas muscle, confirming a diagnosis of iliopsoas haematoma. The case resolved completely after conservative medical treatment in addition to a period of rest and intense active physical therapy. This case study reports the rare diagnosis of an uncomplicated iliopsoas haematoma following a sports injury in a young athlete.

S Afr J Sports Med 2015;27(2):55-57. DOI:10.7196/SAJSM.612

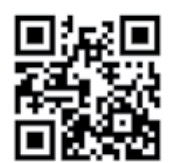

Classified as either spontaneous or traumatic, ${ }^{[1]}$ iliopsoas muscle haematomas are rare lesions that typically appear in individuals presenting with clotting difficulties, whether due to illnesses, such as haemophilia ${ }^{[2]}$ and other blood disorders, or as a result of anticoagulant treatment. ${ }^{[3]}$

Clinical signs and symptoms of iliopsoas haematoma are often vague and nonspecific and may include pelvic, back, lower abdominal, or groin and thigh pain or swelling. Muscle haemorrhage may lead to flexion deformity of the hip and may be associated with the functional inability of the affected limb. ${ }^{[2]}$ Femoral neuropathy due to the compression of the femoral nerve is the most serious and common complication. ${ }^{[2,4]}$

Iliopsoas haematoma is mostly described as an infrequent complication of anticoagulation therapy in older patients, however it has rarely been noted in athletes or in sports-associated incidents. ${ }^{[2,4,5]}$ We report a case that occurred following a closed injury of the iliopsoas muscle in a young rugby player after he was tackled during a game.

\section{Case report}

This report describes a case of a 22 -year-old rugby player who complained of pain in the right hip flexor muscle and lower back areas three days after a rugby match in August 2013. During the first half of the match the player injured his hip when he was tackled and twisted his pelvic area. He was only removed from the field of play later in the game, during the second half, complaining of lower back pain.

On examination directly after the game, he had no pain and full strength with flexion of the right iliopsoas and rectus femoris muscles and no pain on adduction of the right leg. Examination of the lower back was also pain free with a normal range of motion of the vertebral column. His symptoms subsided and no immediate treatment was given.

Three days after the game he complained of excessive pain in the lower back, right hip flexor/pelvic area. On examination he now had pain on palpation of the right hip flexor muscles. There was no pain on resistant flexion movements of both the deep and superficial hip flexor muscles. The examination of the lower back area was still pain free on palpation, but he had some pain with flexion of the vertebral column. He had no pain with the adductor squeeze test or when doing a sit-up. He also had no pain on adduction or abduction of his right leg. The FABER test of the right hip was negative. A possible deep hip flexor muscle strain or lower back injury was suspected. The player was referred for a magnetic resonance imaging (MRI) scan of his lower back and pelvic area.

The MRI lumbar spine scan (1.5 Tesla Siemens Symphony TIM system) revealed oedema in the right psoas muscle, extending from L1 to L5. Centrally in this region of oedema, a more complex area of heterogeneous signal changes of $\sim 3-5 \mathrm{~cm}$ diameter was noted, consistent with subacute haematoma (Fig. 1). The right psoas muscle was also larger than the left (Fig. 2).

A second field of signal changes in the

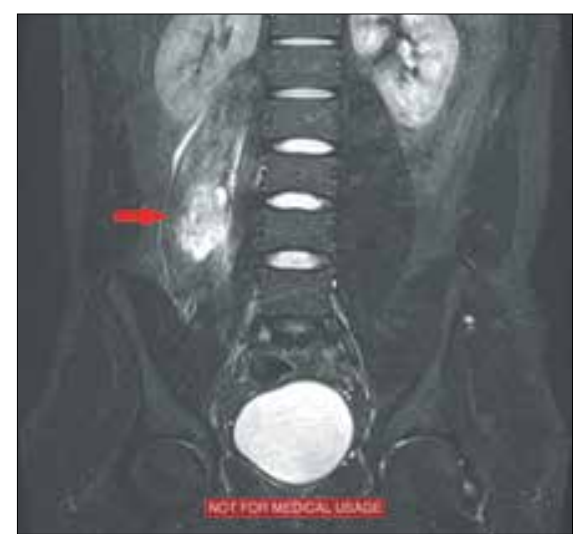

Fig. 1. Short tau inversion recovery (STIR) coronal images demonstrating oedema and circumscribed haematoma in right psoas muscle (red arrow). 


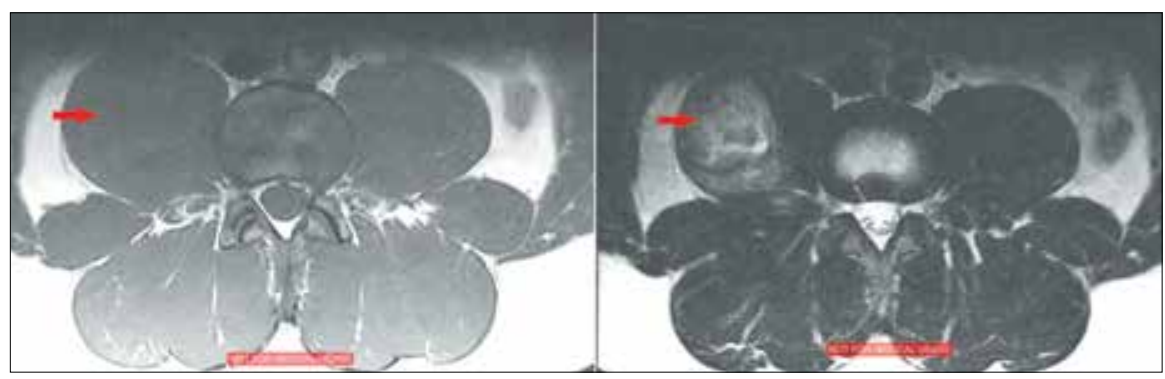

Fig. 2. T1 (left) and T2 (right) axial images depicting a haematoma in the right psoas muscle (red arrow). The right psoas muscle is larger than the left.
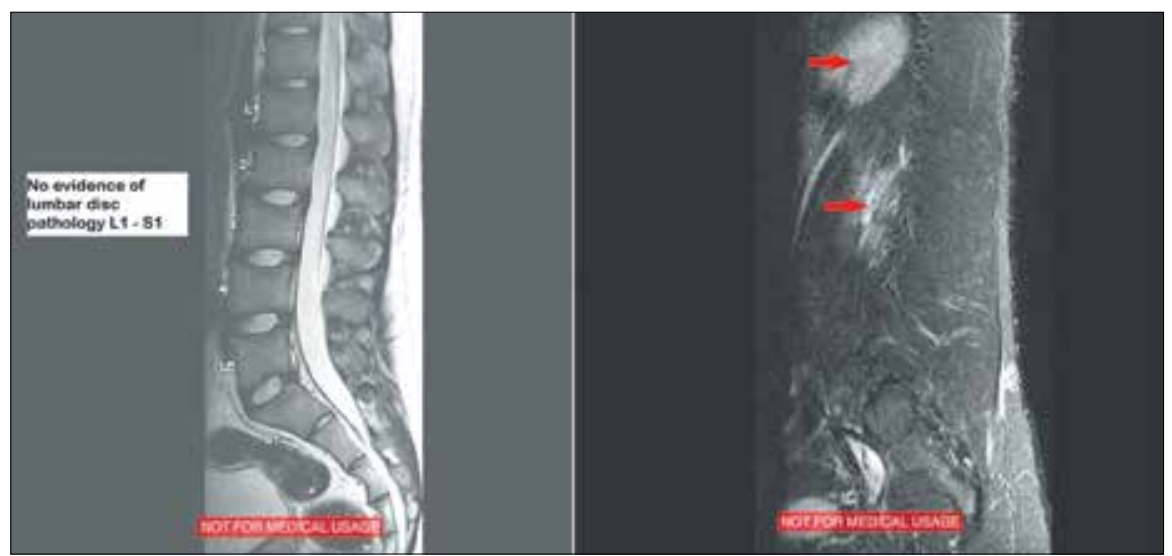

Fig. 3. T2 midsagittal image (left) and STIR right parasagittal image (right), indicating no evidence of lumbar disc pathology, showing oedema in right psoas muscle (red arrows).

right deep gluteal region adjacent to the right greater trochanter was present, representing haemorrhage and oedema in relation and between the gluteus medius and minimus muscles on the right. No evidence of underlying bony involvement or significant lumbar disc pathology was present (Fig. 3).

Treatment was initialised after confirmation of the diagnosis. Treatment consisted of rest from all rugby and conditioning activities for 14 days. The player also received symptomatic physiotherapy treatment consisting of antiinflammatory modalities, and soft tissue mobilisation of muscle around the right hip flexor muscle area. A 10-day course of oral analgesic tablets was prescribed. The patient was pain free on walking and resistant hip flexor muscle movements after 14 days. $\mathrm{He}$ had normal range of motion of the right hip. A gradual running programme was introduced together with resistance training. Sportspecific conditioning commenced after 21 days, and after 28 days he was asymptomatic and returned to full rugby training.

\section{Discussion}

Reports of haematomas of the iliopsoas muscle in young athletes injured during sports events have only rarely been published. $[1,6]$ We report a 22-year-old rugby player that developed an iliopsoas muscle haematoma where the mechanism of injury was most likely excessive muscle strain during a match.

The iliopsoas comprises three muscles passing through the pelvis. The origins are on the lumbar vertebrae (psoas major and psoas minor muscles) and the inner pelvis (iliacus muscle) and inserts into the lesser trochanter on the femur. ${ }^{[4]}$ It is the strongest flexor of the hip joint, and with minimal elastic properties, opposes extension of the hip. These muscles are essential in the support of posture and hip flexion. They work together to flex the hip when kicking, bringing the leg out in front of the body or the knee up towards the chest when running. ${ }^{[2]}$

Mechanisms of injury to the iliopsoas may be due to sudden contraction of the hip flexor muscles while the hip is in a stretched position or during sprinting or kicking activities with the hip extended and stretched above its flexible limit. ${ }^{[7]}$ A sudden movement of the hip into extension from a flexed and externally rotated position, certain movements such as flexion-extension (raising and lowering the entire leg) or rotation (twisting of the hip) may also contribute to an injury. ${ }^{[7]}$

Patients often present with undifferentiated symptoms including sudden onset of severe pain, muscle dysfunction and, in acute cases, with nerve palsy primarily affecting the femoral nerve. ${ }^{[5,8]}$ The flexed position imposes the least tension on the iliopsoas muscle whereby the muscle is relaxed, with pain and limitation on passive hip extension as the muscle is stretched. ${ }^{[9]}$

Owing to the strong fibrous tissue layers of the muscle, large volumes of intramuscular bleeding are entrapped causing severe pain as a result of pressure build-up. Inhibition of muscular function with femoral nerve involvement could present a serious and potentially severe neurological dysfunction. Urgent drainage procedures of a traumatic haematoma may vary from open surgery, ${ }^{[3,8]}$ to percutaneous drainage, ${ }^{[5]}$ laparoscopy ${ }^{[1]}$ or the most recently described retroperitoneoscopic approach. ${ }^{[10]}$ The haematoma itself may account for a fatal outcome because of massive blood loss and haemorrhagic shock, especially with delayed diagnosis. ${ }^{[8]}$ Depending on the rate of active bleeding and the degree of impairment, treatment of an iliopsoas haematoma remains conservative comprising a period of total inactivity, adequate analgesia and gentle physiotherapy to allow the haematoma to spontaneously resorb. ${ }^{[2,3,9]}$

In cases where an iliopsoas haematoma diagnosis is suspected, an MRI scan is the imaging modality of choice due to its high sensitivity and specificity in the detection of small haematomas. ${ }^{[8,9]}$ In patients presenting with femoral neuropathy as a consequence of retroperitoneal bleeding, an MRI scan will assist in ruling out nerve root compression or spinal problems. In view of its usefulness in studying deep soft tissue, ultrasonography, although being operator-dependent and influenced by surrounding organs, may also provide valuable information in detecting these lesions. ${ }^{[6]}$ Computed tomography scans are frequently used in diagnosis as they are more readily accessible. ${ }^{[5,10]}$ Clinical knowledge remains fundamental and a degree of suspicion in the diagnosis is needed due to the insidious incidence of neurological disorders.

Haematoma of the iliopsoas muscle resulting from sport activities is an extraordinary diagnosis. This case in a 22 -year-old rugby player had an uncomplicated course and completely resolved following early detection and diagnosis. 


\section{Consent}

Written informed consent was obtained from the patient for publication of this case report and any accompanying images. A copy of the written consent is available for review.

\section{References}

1. Jing J, Qian J, Tian D, Zhang J, Chen L, Tang J. Laparoscopic treatment of traumatic iliopsoas hematoma. Chin Med J (Engl) 2013;126(4):795-797.

2. Canelles E, Bruna M, Roig JV. Spontaneous hematoma of the iliopsoas muscle: The report of three cases and review of the literature. Rev Esp Cir Ortop Traumatol (English Edition) 2010;54(4):234-237. [http://dx.doi.org/10.1016/S19888856(10)70238-8]

3. Kong WK, Cho K, Lee HJ, Choi J. Femoral neuropathy due to iliacus muscle hematoma in a patient on warfarin therapy. J Korean Neurosurg Soc 2012;51(1):51-53. [http:// dx.doi.org/10.3340/jkns.2012.51.1.51]
4. Tamai K, Kuramochi T, Sakai H, Iwami N, Saotome K. Complete paralysis of the quadriceps muscle caused by traumatic iliacus hematoma: A case report. J Orthop Sci 2002;7(6):713-716. [http://dx.doi.org/10.1007/s007760200127]

5. Patel A, Calfee R, Thakur N, Eberson C. Non-operative management of femoral neuropathy secondary to a traumatic iliacus haematoma in an adolescent. J Bone Joint Surg Br 2008;90(10):1380-1381. [http://dx.doi.org/10.1302/0301-620X.90B10.21040]

6. Kameda T, Fujita M, Takahashi I. Diagnosis of traumatic iliopsoas hematoma using point-of-care ultrasound. Crit Ultrasound J 2011;3(1):59-61. [http://dx.doi.org/10.1007/s13089-011-0065-4]

7. Anderson K, Strickland SM, Warren R. Hip and groin injuries in athletes. Am J Sports Med 2001;29(4):521-533.

8. Conesa X, Ares O, Seijas R. Massive psoas haematoma causing lumbar plexus palsy: A case report. J Orthop Surg (Hong Kong) 2012;20(1):94-97.

9. Basheer A, Jain R, Anton T, Rock J. Bilateral iliopsoas hematoma: Case report and literature review. Surg Neurol Int 2013;4:121-121. [http://dx.doi.org/10.4103/2152-7806.118561]

10. Qian J, Jing J, Tian D, Zhang J, Chen L. Safety and efficacy of a new procedure for treating traumatic iliopsoas hematoma: A retroperitoneoscopic approach. Surg Endosc 2014;28(1):265-270. [http://dx.doi.org/10.1007/s00464-013-3183-1] 